\title{
Melhora dos limiares de sensibilidade do campo visual após redução da pressão intra-ocular em pacientes com glaucoma: tratamento cirúrgico vs. clínico
}

\author{
Improvement in glaucomatous visual field thresholds after reduction of \\ intraocular pressure: clinical vs. surgical treatment
}

\author{
Leopoldo Magacho ${ }^{1}$ \\ Carlos Frederico Queiroz ${ }^{2}$ \\ Mariele Medeiros ${ }^{3}$ \\ Francisco Eduardo Lima ${ }^{4}$ \\ Bernardo Magacho5 \\ Marcos Ávila ${ }^{6}$
}

Trabalho realizado no Centro de Referência em Oftalmologia (CEROF) - Universidade Federal de Goiás - UFG - Goiânia (GO) - Brasil.

${ }^{1}$ Doutor em Oftalmologia pela Universidade de Campinas - UNICAMP - Campinas (SP) - Brasil.

${ }^{2}$ Ex-fellow do Setor de Glaucoma do CEROF da UFG Goiânia (GO) - Brasil.

${ }^{3}$ Ex-fellow do Setor de Glaucoma do CEROF da UFG Goiânia (GO) - Brasil.

${ }^{4}$ Doutor em Oftalmologia pela Universidade de São Paulo - USP - São Paulo (SP).

${ }^{5}$ Residente do CEROF da UFG - Goiânia (GO) - Brasil.

${ }^{6}$ Professor Doutor e Chefe do CEROF da UFG - Goiânia (GO) - Brasil.

Endereço para correspondência: Leopoldo Magacho. Rua 131, nº 313 - Setor Sul - Goiânia (GO) CEP 74093-200 E-mail: lmagacho@brturbo.com

Recebido para publicação em 04.04.2005

Versão revisada recebida em 01.08.2005

Aprovação em 06.11.2005

\begin{tabular}{|c|}
\hline RESUMO \\
\hline 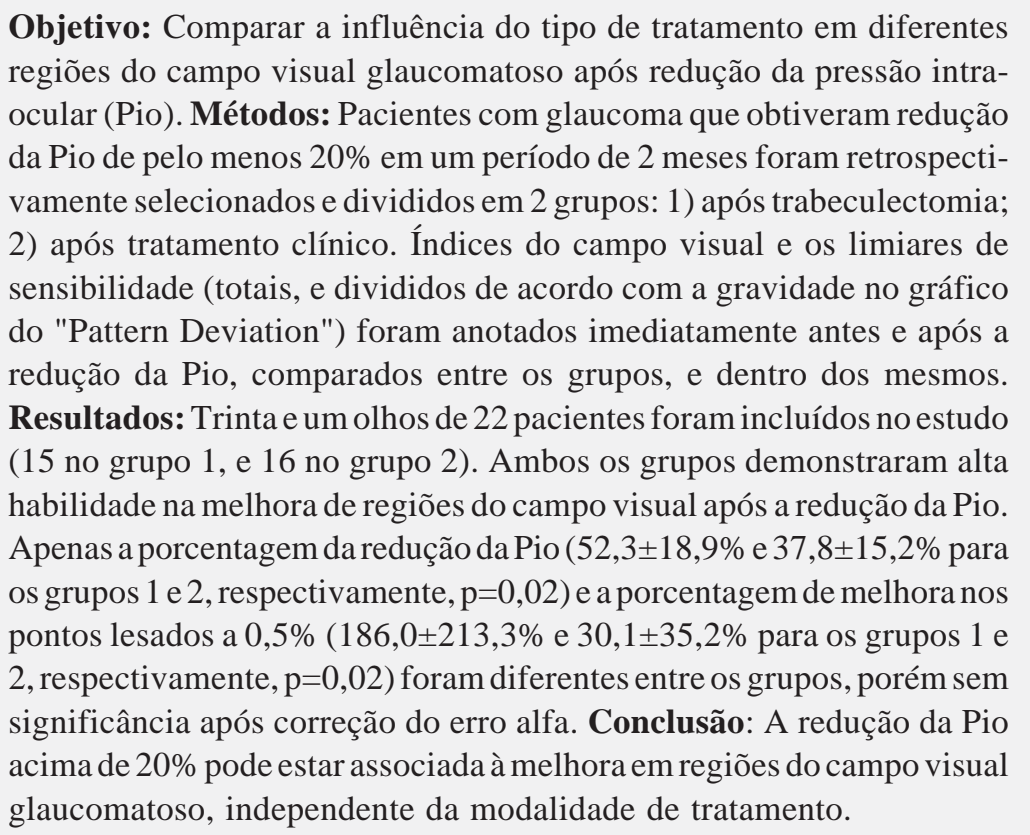 \\
\hline
\end{tabular}

Descritores: Pressão intra-ocular; Glaucoma; Perimetria; Tonometria ocular; Estudos retrospectivos; Resultado de tratamento

\section{INTRODUÇÃO}

O glaucoma é caracterizado por dano progressivo e irreversível ao disco óptico e camada de fibras nervosas da retina (CFNR). A pressão intraocular (Pio) pode ser considerada como o principal fator de risco para o glaucoma $^{(1)}$, e sua redução como o único meio efetivo de tratamento ${ }^{(2)}$, incluindo pacientes com glaucoma de pressão normal ${ }^{(3)}$.

Estudos têm demonstrado melhora na $\mathrm{CFNR}^{(4-5)}$, no aspecto do disco óptico $^{(6-8)}$ e no campo visual glaucomatoso ${ }^{(8-14)}$ após a redução da Pio após a trabeculectomia. Portanto, é racional sugerir que a redução medicamentosa da Pio também conduziria a uma melhora destas estruturas. $\mathrm{O}$ objetivo deste estudo é verificar a influência da modalidade de tratamento nos limiares de sensibilidade do campo visual glaucomatoso após a redução da Pio. 


\section{MÉTODOS}

Este foi um estudo retrospectivo. Os prontuários de todos os pacientes glaucomatosos avaliados por um dos autores (FEL) em um período de 2 meses (janeiro e fevereiro de 2001) com história de redução da Pio em no mínimo $20 \%$ foram considerados. Além disso, era necessário pelo menos 3 exames de campo visual anteriores ao tratamento. Os pacientes foram subseqüentemente divididos em dois grupos: redução cirúrgica (RC) e redução medicamentosa (RM) da Pio.

Os critérios de inclusão para ambos os grupos foram: pacientes com diagnóstico de glaucoma primário de ângulo aberto (GPAA) (Pio > $21 \mathrm{mmHg}$, aferida com tonômetro de Goldmann, gonioscopia evidenciando ângulo aberto ${ }^{(15)}$, e neuropatia óptica glaucomatosa, definida como a presença de perda localizada ou global da rima neural, hemorragia de disco óptico ou assimetria escavação/disco > 0,2). Além disso, um defeito glaucomatoso característico no campo visual glaucomatoso $^{(16)}$ precisava estar presente em no mínimo dois exames confiáveis $^{(17)}$ Sita Standard 24-2 (Humphrey Systems, Dublin, CA, EUA) antes da redução da Pio. Olhos afácicos e pseudofácicos e pacientes de ambos os grupos que apresentaram qualquer alteração biomicroscópica ou fundoscópica que pudesse interferir com os exames (opacidades corneanas, cataratas significantes ${ }^{(18)}$, alterações maculares, etc) foram excluídos.

Apenas pacientes com trabeculectomia primária simples (sem facectomia associada) foram incluídos. O procedimento foi executado por um único e experiente cirurgião (FEL) com base limbo, retalho escleral triangular e mitomicina $\mathrm{C} 0,5 \mathrm{mg} / \mathrm{ml}$ por 3 minutos. Os pacientes cirúrgicos foram excluídos se houvesse qualquer complicação, como hipotonia (definida no presente estudo como Pio $<6 \mathrm{mmHg}$ em qualquer avaliação pós-operatória), perda vítrea, descolamento de retina ou coróide. No grupo $\mathrm{RC}$ os pacientes foram excluídos caso a pressão alvo não tenha sido atingida sem auxílio medicamentoso. No grupo RM todas as drogas foram consideradas, com exceção à pilocarpina.

Os dados foram analisados pelo software SPSS (SPSS Inc, Chicago, IL, EUA) versão 10.1. Dois campos visuais consecutivos, o último antes da redução da Pio e o primeiro após, com intervalo de não mais que 6 meses do tratamento foram incluídos na análise. Os índices globais do campo visual "Mean Deviation" (MD) e "Pattern Standard Deviation" (PSD) e os valores dos limiares (médias) no gráfico do "Pattern Deviation" (PD) foram comparados entre os grupos. Além disso, em uma segunda análise, foi realizado subdivisões, considerando os pontos do gráfico PD como: normal (melhor que 5\% no PD), alterado (todos os pontos alterados $\leq 5 \%$ ), pontos de $2 \%$ e $5 \%$, pontos de $1 \%$ e $0,5 \%$ e apenas pontos de $0,5 \%$ (médias dos valores de cada subgrupo) para testar se havia alguma diferença na melhora do campo visual de acordo com a severidade do campo visual. A análise dos grupos foi feita ponto-aponto tendo como base as regiões lesadas no campo visual antes da redução da Pio. Dessa forma, esses pontos eram mantidos naquele subgrupo, independentemente da sua classificação estatística após a redução da Pio, tornando a análise mais fidedigna. Isso ocorreu porque um determinado ponto poderia melhorar ou piorar após tratamento, saindo do seu grupo inicial.

O teste de Komogorov-Smirnov foi utilizado para se testar a normalidade das amostras (distribuição Gaussiana ou não). Os grupos foram comparados com o teste t de Student independente. A porcentagem da melhora para todos os valores foi comparada pelo mesmo teste. $\mathrm{O}$ teste $\mathrm{T}$ pareado foi usado para comparar os índices do campo visual entre os grupos. Finalmente, a correlação de Pearson foi utilizada para testar a correlação entre a porcentagem da redução da Pio e a porcentagem da melhora do campo visual nos grupos. A correção do erro alfa foi realizada segundo o modelo proposto por Cross e Chaffin ${ }^{(19)}$. Dessa forma, valores de "p" menores que 0,0125 foram considerados significantes para os cálculos entre os grupos e $\mathrm{p}<0,02$ para cálculos dentro dos grupos. Nos dados categóricos, $\mathrm{p}<0,05$ foi considerado como estatisticamente significativo. Ao se considerar o erro alfa acima, a diferença das médias a ser detectada de 1 , bem como o seu desvio padrão, o poder do teste foi de $60 \%$ para os cálculos entre grupos e de $63 \%$ dentro dos mesmos.

\section{RESULTADOS}

Trinta e um olhos de 22 pacientes foram incluídos no estudo (15 no grupo RC e 16 no grupo RM). Não houve exclusão de qualquer paciente no grupo cirúrgico. A idade média do grupo RM $(66,7 \pm 11,8$ anos) foi significativamente maior do que o grupo $\mathrm{RC}(57,9 \pm 8,7$ anos, $\mathrm{p}=0,02)$. Os dados do campo visual, com as comparações entre os grupos antes e depois da redução da Pio estão dispostos na tabela 1 .

Ambos os grupos demonstraram melhora em vários parâmetros do campo visual após a redução da Pio (Tabela 2). Comparando os grupos em relação à porcentagem da redução dos índices globais do campo visual, e a porcentagem da melhora dos pontos do PD, apenas a redução da Pio e a melhora dos pontos $0,5 \%$ mostraram inicialmente diferença estatística, o que não aconteceu após correção do erro alfa (Tabela 3). A correlação de Pearson mostrou que apenas a porcentagem de melhora nos pontos $1 \%$ e $0,5 \%$ foram estatisticamente correlacionadas com a porcentagem de redução da Pio no grupo $\mathrm{RC}(\mathrm{r}=0,772, \mathrm{p}=0,01)$. Nenhuma variável foi correlacionada com a redução da Pio no grupo RM (Tabela 4).

\section{DISCUSSÃO}

Apesar do reconhecimento de que a Pio é apenas um fator de risco para o glaucoma, sua redução ainda é considerada essencial no controle da doença. Estudos têm demonstrado melhora dos tecidos oculares após a redução da Pio em pacientes com glaucoma e em diferentes regiões do campo visual ${ }^{(4-14,20-21)}$. É importante enfatizar, entretanto, que todos os estudos foram baseados na trabeculectomia como o método 


\begin{tabular}{|c|c|c|c|c|c|}
\hline & Grupo* & Antes da redução da Pio & $\mathbf{P}^{*}$ & Após a redução da Pio & $\mathbf{P}^{*}$ \\
\hline $\mathrm{MD}(\mathrm{dB})$ & $\begin{array}{l}\mathrm{RC} \\
\mathrm{RM}\end{array}$ & $\begin{array}{l}-8,9 \pm 9,1 \\
-4,9 \pm 3,8\end{array}$ & 0,1 & $\begin{array}{l}-7,8 \pm 9,3 \\
-4,9 \pm 4,2\end{array}$ & 0,3 \\
\hline PSD (dB) & $\begin{array}{l}\mathrm{RC} \\
\mathrm{RM}\end{array}$ & $\begin{array}{l}5,2 \pm 3,2 \\
4,5 \pm 2,6\end{array}$ & 0,4 & $\begin{array}{l}4,2 \pm 2,6 \\
4,1 \pm 2,7\end{array}$ & 0,9 \\
\hline Pio $(\mathrm{mmHg})$ & $\begin{array}{l}\mathrm{RC} \\
\mathrm{RM}\end{array}$ & $\begin{array}{l}26,2 \pm 8,1 \\
21,6 \pm 7,1\end{array}$ & 0,1 & $\begin{array}{l}11,3 \pm 2,9 \\
12,5 \pm 1,8\end{array}$ & 0,2 \\
\hline Todos os pontos lesados (dB) & $\begin{array}{l}\mathrm{RC} \\
\mathrm{RM}\end{array}$ & $\begin{array}{l}16,1 \pm 9,7 \\
18,3 \pm 4,6\end{array}$ & 0,4 & $\begin{array}{l}20,1 \pm 10,2 \\
21,1 \pm 5,4\end{array}$ & 0,7 \\
\hline Pontos sem lesão (dB) & $\begin{array}{l}\mathrm{RC} \\
\mathrm{RM}\end{array}$ & $\begin{array}{l}21,6 \pm 8,5 \\
25,1 \pm 2,1\end{array}$ & 0,1 & $\begin{array}{l}21,9 \pm 8,8 \\
24,5 \pm 2,7\end{array}$ & 0,2 \\
\hline Pontos lesados a 2 e $5 \%(\mathrm{~dB})$ & $\begin{array}{l}\mathrm{RC} \\
\mathrm{RM}\end{array}$ & $\begin{array}{l}16,8 \pm 8,3 \\
19,6 \pm 3,9\end{array}$ & 0,2 & $\begin{array}{l}20,1 \pm 9,9 \\
22,4 \pm 5,1\end{array}$ & 0,4 \\
\hline Pontos lesados a 1 e $0,5 \%(\mathrm{~dB})$ & $\begin{array}{l}\mathrm{RC} \\
\mathrm{RM}\end{array}$ & $\begin{array}{l}13,5 \pm 8,8 \\
18,3 \pm 6,1\end{array}$ & 0,1 & $\begin{array}{l}17,7 \pm 10,6 \\
20,5 \pm 7,1\end{array}$ & 0,4 \\
\hline Pontos lesados a $0,5 \%(\mathrm{~dB})$ & $\begin{array}{l}\mathrm{RC} \\
\mathrm{RM}\end{array}$ & $\begin{array}{l}12,0 \pm 10,0 \\
14,3 \pm 6,5\end{array}$ & 0,5 & $\begin{array}{l}18,7 \pm 8,2 \\
18,4 \pm 8,6\end{array}$ & 0,9 \\
\hline $\begin{array}{l}\text { Tempo em meses entre o } \\
\text { campo visual e a redução da Pio }\end{array}$ & $\begin{array}{l}\mathrm{RC} \\
\mathrm{RM}\end{array}$ & $\begin{array}{l}1,3 \pm 0,6 \\
3,5 \pm 1,8\end{array}$ & $<0,001$ & $\begin{array}{l}4,6 \pm 0,8 \\
4,5 \pm 0,9\end{array}$ & 0,6 \\
\hline
\end{tabular}

\begin{tabular}{|lcc|}
\hline \multicolumn{4}{|c|}{$\begin{array}{c}\text { Tabela 2. Comparação das variáveis do campo visual após a redu- } \\
\text { ção da Pio nos grupos }\end{array}$} \\
RC & $\mathbf{R M}^{*}$ \\
MD (dB) & 0,002 & 0,9 \\
PSD (dB) & 0,06 & 0,2 \\
Pio (dB) & $<0,001$ & 0,001 \\
Todos os pontos lesados (dB) & 0,003 & 0,004 \\
Pontos sem lesão (dB) & 0,4 & 0,07 \\
Pontos lesados a 2 e 5\% (dB) & $<0,001$ & 0,006 \\
Pontos lesados a 1 e 0,5\% (dB) & 0,001 & 0,1 \\
Pontos lesados a 0,5\% (dB) & 0,04 & 0,02 \\
* Teste de t pareado & & \\
Pio=pressão intra-ocular; RC=redução cirúrgica; $R M=$ redução medicamentosa \\
\hline
\end{tabular}

de redução da Pio. Entretanto, este procedimento cirúrgico não é empregado em todo paciente com glaucoma que necessita controle da Pio. A redução medicamentosa da Pio é provavelmente o método mais utilizado. Portanto, torna-se fundamental avaliar sua influência nos tecidos que são lesados no glaucoma.

Em nosso estudo, os resultados dos campos visuais foram estatisticamente similares entre os grupos. Conseqüentemente, acreditamos que estamos simplesmente comparando ambos os grupos em relação à modalidade de tratamento e também segundo a redução da Pio, estatisticamente diferente entre os grupos (Tabela 3).

A melhora no campo visual após trabeculectomia (média de redução da Pio: 47,5\% nos campos que demonstram melhora contra $40,0 \%$ naqueles sem melhora, $\mathrm{p}=0,7)$ é maior nas regiões com os menores níveis de sensibilidade antes da redução da Pio ${ }^{(13)}$. Esses autores também postularam que os subgrupos com os melhores níveis de sensibilidade estão associados com células ganglionares predominantemente sadias, e conseqüentemente a probabilidade de melhora é menor. No presente estudo, a redução da Pio de 52,3 $\pm 18,9 \%$ no grupo RC e $37,8 \pm 15,2 \%$ no grupo RM foi associada com a melhora nos pontos do campo visual com dano prévio (Tabela 2, $\mathrm{p}=0,03 \mathrm{e}$ $\mathrm{p}=0,04$, respectivamente), entretanto não significativo após a correção alfa. Somando-se a isto, ambos os grupos demonstraram melhora em regiões do campo visual com diferentes níveis de sensibilidade (Tabela 2).

Entretanto, Salim et al. tinham categorizado os campos visuais de acordo com os pontos com sensibilidade maiores $(25 \%)$, menores $(25 \%)$ e intermediários $(50 \%)^{(13)}$. É possível existir campos visuais severos em que os níveis mais altos de sensibilidade estão classificados como $1 \%$ ou $0,5 \%$. Além disso, é possível a existência de campos visuais iniciais em que os níveis menores que $25 \%$ estariam danificados, em média, a 5\%. Portanto, uma classificação de limiares que utiliza o mapa de probabilidade do Humphrey, como utilizado no presente estudo, ilustraria uma idéia mais precisa do dano real às células ganglionares. Além disso, é possível que diferentes métodos de análise de progressão do campo visual pudessem gerar resultados variados, como já demonstrado anteriormen$\mathrm{te}^{(22)}$. Dessa forma, a análise ponto-a-ponto tendo como base a manutenção original dos pontos no campo visual lesado antes da redução da Pio exclui essa possível fonte de viés.

Em um estudo clínico multicêntrico randomizado (Collaborative Initial Glaucoma Treatment Study) avaliou a longo prazo pacientes submetidos à redução da Pio pela trabeculectomia ou tratamento clínico e os comparou ${ }^{(23)}$. O campo visual não diferenciou muito do início do tratamento. Apesar da maior perda de campo visual inicial do grupo cirúrgico (principalmente pelo surgimento ou piora da catarata), essa diferença não foi encontrada após cerca de 5 anos de acompanhamento. É possível que a forma de análise de progressão do campo 


\begin{tabular}{|c|c|c|c|}
\hline${ }^{\dagger}$ Porcentagem de: & Grupo & $(\%)$ & $\mathbf{p}^{*}$ \\
\hline Redução da Pio & $\begin{array}{l}\mathrm{RC} \\
\mathrm{RM}\end{array}$ & $\begin{array}{l}52,3 \pm 18,9 \\
37,8 \pm 15,2\end{array}$ & 0,02 \\
\hline Redução no MD & $\begin{array}{l}\mathrm{RC} \\
\mathrm{RM}\end{array}$ & $\begin{array}{r}25,1 \pm 25,3 \\
8,7 \pm 37,6\end{array}$ & 0,1 \\
\hline Redução no PSD & $\begin{array}{l}\mathrm{RC} \\
\mathrm{RM}\end{array}$ & $\begin{array}{r}10,5 \pm 35,6 \\
9,2 \pm 24,7\end{array}$ & 0,9 \\
\hline Melhora em todos os pontos lesados & $\begin{array}{l}\mathrm{RC} \\
\mathrm{RM}\end{array}$ & $\begin{array}{l}40,8 \pm 44,1 \\
16,1 \pm 19,6\end{array}$ & 0,07 \\
\hline Melhora em todos os pontos estatisticamente normais & $\begin{array}{l}\mathrm{RC} \\
\mathrm{RM}\end{array}$ & $\begin{array}{cc}1,99 \pm & 7,1 \\
-2,5 \pm & 5,0\end{array}$ & 0,05 \\
\hline Melhora nos pontos lesados a $2 \%$ e $5 \%$ & $\begin{array}{l}\mathrm{RC} \\
\mathrm{RM}\end{array}$ & $\begin{array}{l}19,9 \pm 12,2 \\
14,2 \pm 16,9\end{array}$ & 0,3 \\
\hline Melhora nos pontos lesados a $1 \%$ e $0,5 \%$ & $\begin{array}{l}\mathrm{RC} \\
\mathrm{RM}\end{array}$ & $\begin{array}{l}46,1 \pm 50,3 \\
19,1 \pm 44,4\end{array}$ & 0,2 \\
\hline Melhora nos pontos lesados a $0,5 \%$ & $\begin{array}{l}\mathrm{RC} \\
\mathrm{RM}\end{array}$ & $\begin{array}{r}186,0 \pm 213,3 \\
30,1 \pm 35,2\end{array}$ & 0,02 \\
\hline
\end{tabular}

\begin{tabular}{|c|c|c|c|}
\hline \multicolumn{4}{|c|}{$\begin{array}{l}\text { Tabela 4. Correlação entre a porcentagem de redução da pressão } \\
\text { intra-ocular e a porcentagem de melhora de áreas do campo visual } \\
\text { com diferentes limiares de sensibilidade }\end{array}$} \\
\hline †Porcentagem de: & Grupo & $\begin{array}{l}\text { Correlação } \\
\text { de Pearson }\end{array}$ & $p$ \\
\hline Redução no MD & $\begin{array}{l}\mathrm{RC} \\
\mathrm{RM}\end{array}$ & $\begin{array}{r}0,124 \\
-0,153\end{array}$ & $\begin{array}{l}0,6 \\
0,5\end{array}$ \\
\hline Redução no PSD & $\begin{array}{l}\mathrm{RC} \\
\mathrm{RM}\end{array}$ & $\begin{array}{r}-0,123 \\
0,352\end{array}$ & $\begin{array}{l}0,6 \\
0,1\end{array}$ \\
\hline $\begin{array}{l}\text { Melhora em todos } \\
\text { os pontos lesados }\end{array}$ & $\begin{array}{l}\mathrm{RC} \\
\mathrm{RM}\end{array}$ & $\begin{array}{r}0,408 \\
-0,186\end{array}$ & $\begin{array}{l}0,1 \\
0,4\end{array}$ \\
\hline $\begin{array}{l}\text { Melhora em todos os pontos } \\
\text { estatisticamente normais }\end{array}$ & $\begin{array}{l}\mathrm{RC} \\
\mathrm{RM}\end{array}$ & $\begin{array}{r}0,110 \\
-0,308\end{array}$ & $\begin{array}{l}0,6 \\
0,2\end{array}$ \\
\hline $\begin{array}{l}\text { Melhora nos pontos } \\
\text { lesados a } 2 \% \text { e } 5 \%\end{array}$ & $\begin{array}{l}\mathrm{RC} \\
\mathrm{RM}\end{array}$ & $\begin{array}{r}0,369 \\
-0,111\end{array}$ & $\begin{array}{l}0,2 \\
0,6\end{array}$ \\
\hline $\begin{array}{l}\text { Melhora nos pontos } \\
\text { lesados a } 1 \% \text { e } 0,5 \%\end{array}$ & $\begin{array}{l}\mathrm{RC} \\
\mathrm{RM}\end{array}$ & $\begin{array}{l}0,772 \\
0,301\end{array}$ & $\begin{array}{l}0,01 \\
0,3\end{array}$ \\
\hline † Considerando a média dos valore & & & \\
\hline
\end{tabular}

visual, como comentado anteriormente, seja responsável por essas diferenças em relação ao presente estudo.

No presente estudo, ambos os grupos mostraram melhora em pontos do campo visual com diferentes limiares de sensibilidade (Tabela 2). A única exceção foram os pontos lesados a $1 \%$ e $0,5 \%$ no grupo RM. Uma possível explicação está relacionada com o " $n$ " da amostra estudada, diminuindo o poder do teste.

Na média, todos os pontos mostraram uma melhora significante dos limiares de sensibilidade após a trabeculectomia. Acreditamos que a falta de diferença estatística está relacionada com o tamanho da amostra do teste. A melhora foi melhor verificada nos pontos mais lesados $(1 \%$ e $0,5 \%)$, os únicos que tiveram alta correlação com a redução da Pio no grupo $\mathrm{RC}(\mathrm{r}=0,772, \mathrm{p}=0,01)$. Possivelmente amostras maiores esclareceriam a real importância da porcentagem da redução da Pio na redução dos limiares do campo visual em pacientes glaucomatosos.

Um outro fator de confusão pode estar relacionado ao uso de medicações, já que, freqüentemente após a cirurgia, o paciente utiliza uma quantidade menor de medicações antiglaucomatosas. Os efeitos colaterais destas medicações, em particular a pilocarpina, podem ser responsáveis por visão borrada e redução de sensibilidade do campo visual ${ }^{(24)}$. Entretanto, usuários de pilocarpina foram excluídos da análise, e outras medicações geralmente não reduzem o tamanho pupilar. Além disso, a própria trabeculectomia pode ter efeito na sensibilidade do campo visual. Uma possível formação de catarata após a trabeculectomia pode ser responsável por alterações nos limiares do campo visual. Apesar de termos utilizado o "Pattern Deviation" em nossa análise, que pode filtrar a influência da catarata no campo visual, a influência da opacidade de meios pode influenciar outros parâmetros avaliados, como o $\mathrm{MD}^{(17)}$.

Outra limitação do estudo foi a restrição da análise a apenas dois campos visuais por paciente. Flutuações em longo prazo e efeito aprendizado podem também influenciar os limiares do campo visual. Uma análise comparativa de vários campos visuais poderia limitar este tipo de viés.

\section{CONCLUSÃO}

Pode-se concluir que a redução da Pio pode estar associada à melhora dos limiares alterados do campo visual, independente da modalidade do tratamento. Maiores níveis de redução da Pio pode estar relacionado à melhora nos pontos com menores níveis de sensibilidade em pacientes com glaucoma. Estudos prospectivos com uma amostra maior são necessários para se confirmar os resultados aqui encontrados.

\section{ABSTRACT}

Purpose: To verify the influence of modality of treatment in regions of the glaucomatous visual field after reduction of 
intraocular pressure (IOP). Methods: Glaucoma patients that had undergone a reduction of IOP of at least $20 \%$ in a period of 2 months were retrospectively selected and divided into 2 groups: 1) after trabeculectomy; 2) after clinical treatment. Visual field global indices and thresholds (average, divided according to the severity based on the Pattern Deviation plot) were compared before and after IOP reduction. Results: Thirty-one eyes of 22 patients were selected (15 in group 1, and 16 in group 2). Both groups showed good ability regarding improvement of different regions of the visual field after IOP reduction. Only the percentage of reduction of IOP $(52.3 \pm 18.9 \%$ and $37.8 \pm 15.2 \%$ for groups 1 and 2 , respectively, $\mathrm{p}=0.02$ ) and the percentage of improvement in the points damaged at $\mathrm{p}<0.5 \%(186.0 \pm 213.3 \%$ and $30.1 \pm 35.2 \%$ for groups 1 and 2 , respectively, $p=0.02$ ) were different, but not statistically significant after alpha error correction between groups. Conclusion: The reduction of IOP above $20 \%$ may be associated with improvement in regions of the glaucomatous visual field, regardless of treatment modality.

Keywords: Intraocular pressure; Glaucoma; Perimetry; Tonometry, ocular; Retrospective studies; Treatment outcome

\section{REFERÊNCIAS}

1. Sommer A, Tielsch JM, Katz J, Quigley HA, Gottsch JD, Javitt J, Singh K. Relationship between intraocular pressure and primary open angle glaucoma among white and black Americans. The Baltimore Eye Survey. Arch Ophthalmol. 1991;109(8):1090-5.

2. The Advanced Glaucoma Intervention Study (AGIS): 7. The relationship between control of intraocular pressure and visual field deterioration. The AGIS Investigators. Am J Ophthalmol. 2000;130(4):429-40.

3 . The effectiveness of intraocular pressure reduction in the treatment of normaltension glaucoma. Collaborative Normal-Tension Glaucoma Study Group. Am J Ophthalmol. 1998;126(4):498-505.

4. Yamada N, Tomita G, Yamamoto T, Kitazawa Y. Changes in the nerve fiber layer thickness following a reduction of intraocular pressure after trabeculectomy. J Glaucoma. 2000;9(5):371-5.

5. Sogano S, Tomita G, Kitazawa Y. Changes in retinal nerve fiber layer thickness after reduction of intraocular pressure in chronic open-angle glaucoma. Ophthalmology. 1993;100(8):1253-8.
6. Lesk MR, Spaeth GL, Azuara-Blanco A, Araujo SV, Katz LJ, Terebuh AK, et al. Reversal of optic disc cupping after glaucoma surgery analyzed with a scanning laser tomograph. Ophthalmology. 1999;106(5):1013-8.

7. Pederson JE, Herschler J. Reversal of glaucomatous cupping in adults. Arch Ophthalmol. 1982;100(3):426-31.

8. Katz LJ, Spaeth GL, Cantor LB, Poryzees EM, Steinmann WC. Reversible optic disk cupping and visual field improvement in adults with glaucoma. Am J Ophthalmol. 1989;107(5):485-92.

9. Gandolfi SA. Improvement of visual field indices after surgical reduction of intraocular pressure. Ophthalmic Surg. 1995;26(2):121-6.

10. Matsubara K, Maeda M, Tomita G, Kitazawa Y. [The effect of the intraocular pressure reduction on glaucomatous disc changes and visual field defects]. Nippon Ganka Gakkai Zasshi. 1992;96(2):217-24. Japanese.

11. Yildirim E, Bilge AH, Ilker S. Improvement of visual field following trabeculectomy for open angle glaucoma. Eye. 1990;4(Pt 1):103-6.

12. Vuori ML, Vainio-Jylha E, Viitanen TT. Localized changes in glaucomatous visual fields after trabeculectomy. Acta Ophthalmol Scand. 2001;79(5):468-71.

13. Salim S, Paranhos A, Lima M, Shields MB. Influence of surgical reduction of intraocular pressure on regions of the visual field with different levels of sensitivity. Am J Ophthalmol. 2001;132(4):496-500.

14. Spaeth GL. The effect of change in intraocular pressure on the natural history of glaucoma: lowering intraocular pressure in glaucoma can result in improvement of visual fields. Trans Ophthalmol Soc U K. 1985;104(Pt 3):256-64.

15. Shaffer RN. Primary glaucomas. Gonioscopy, ophthalmoscopy and perimetry. Trans Am Acad Ophthalmol Otolaryngol. 1960;64:112-27.

16. Hodapp E, Parrish II RK, Anderson DR. Clinical decisions in glaucoma. St. Louis: Year Book; 1993.

17. Anderson DR, Patella VM. Automated static perimetry. 2nd ed. St. Louis: Mosby; 1999.

18. Chylack LT Jr, Wolfe JK, Singer DM, Leske MC, Bullimore MA, Bailey IL, et al. The Lens Opacities Classification System III. The Longitudinal Study of Cataract Study Group. Arch Ophthalmol. 1993;111(6):831-6.

19. Cross EM, Chaffin WW. Use of the binomial theorem in interpreting results of multiple tests of significance. Educat Psychol Measure. 1982;42(1):25-34.

20. Greenidge KC, Spaeth GL, Traverso CE. Change in appearance of the optic disc associated with lowering of intraocular pressure. Ophthalmology. 1985;92(7): 897-903.

21. Tsai CS, Shin DH, Wan JY, Zeiter JH. Visual field global indices in patients with reversal of glaucomatous cupping after intraocular pressure reduction. Ophthalmology. 1991;98(9):1412-9.

22. Vesti E, Johnson CA, Chauhan BC. Comparison of different methods for detecting glaucomatous visual field progression. Invest Ophthalmol Vis Sci. 2003;44(9):3873-9.

23. Lichter PR, Musch DC, Gillespie BW, Guire KE, Janz NK, Wren PA, Mills RP, CIGTS Study Group. Interim clinical outcomes in the Collaborative Initial Glaucoma Treatment Study comparing initial treatment randomized to medications or surgery. Ophthalmology. 2001;108(11):1943-53.

24. Lindenmuth KA, Skuta GL, Rabbani R, Musch DC. Effects of pupillary constriction on automated perimetry in normal eyes. Ophthalmology. 1989;96(9): 1298-301. 